\title{
Les différentes politiques d'apport en vitamines en Europe et dans le monde (États-Unis)
}

\section{The various policies on vitamin intake in Europe and worldwide (United States)}

Oléagineux, Corps Gras, Lipides. Volume 7, Numéro 3, 280-6, Mai - Juin 2000, Dossier : Les vitamines liposolubles

\section{Auteur(s) : Geneviève POTIER DE COURCY}

Résumé : Les recommandations concernant les apports en vitamines sont définies régulièrement dans les principaux pays occidentaux par des comités d'experts, selon des critères fondés d'une part sur des données scientifiques, et d'autre part sur un certain nombre d'éléments liés à la "politique » nutritionnelle et agro-alimentaire propre à chacun de ces pays. En France, ces apports recommandés ont pris le nom d'apports nutritionnels conseillés ou ANC : deux éditions de ces recommandations " pour la population française » ont vu le jour successivement en 1989 et en 1992 ; la prochaine devrait sortir avant la fin de cette année 2000.

Summary: The need for norms in the supply of (micro) nutriments has gradually become apparent, alongside anxiety from a growing number of users (consumers, manufacturers, scientists and politicians) about the excess consumption of certains nutriments, consumer concern about food, and competition between industrialists and between countries. These norms have taken the form of a series of benchmark values, particularly with regard to vitamins, going from a lower limit defining the dangers of deficiencies up to the safe limit and maximum intake defining the dangers (or absence thereof) of excess, with recommended daily intake per person in between (a list of French recommended daily intakes was published in 2000). The latter take account of scientific data on the prevention of major pathologies and may therefore be regarded as optimum. Despite efforts to bring these recommendations into line, particularly through Codex Alimentarius and the EU, with a view to greater trading and health efficiency, they may vary from one country to another. This is because the policies on recommended daily intake in a particular country are the result of a variety of factors: experts' opinions, the validity of scientific knowledge, pressure from industrialists, changing fashions, \pm "natural" tastes and behaviour on the part of consumers, level of concern about public health and the nutritional level of the population as a whole. In order to correct any anomalies in the supply of vitamins to their populations, the various countries use, depending on the situation, the following methods: making the addition of vitamins to certain foods obligatory (margarine, cereals), allowing their addition to other foods by manufacturers (see feature), putting them back into other foods (milk), awareness campaigns..., but always within a legal framework.

Keywords: needs, recommendations, dangers, addition, public health. 


\section{ARTICLE}

\section{Présentation générale}

La liste des utilisateurs de ces ANC, détaillée dans la précédente édition [1], est la suivante :

- les épidémiologistes et nutritionnistes ;

- les responsables de centres de restauration collective sociale ou privée ;

- les concepteurs de « rations-types » destinées aux forces armées, alpinistes, navigateurs... ;

- les industriels de l'agroalimentaire soucieux de promouvoir la qualité nutritionnelle de leurs produits

- les médecins et les diététiciens dans leurs conseils à leurs patients, [...] et les " professions-relais » : enseignants, pharmaciens, journalistes... ;

- les responsables nationaux de la politique alimentaire et nutritionnelle dans : l'observation des rapports entre les niveaux de consommation et la santé publique, l'élaboration de leurs actions économiques sur le long terme ;

- les professionnels dans l'étiquetage nutritionnel de leurs produits dans l'attente de l'harmonisation européenne de 1993 ;

- en outre, et enfin, « il est souhaitable de faire prendre conscience aux Français de leur responsabilité dans le maintien de leur santé et de celle des membres de leur famille. [...] À côté de publications scientifiques qui font état des recherches en cours et qui portent souvent sur des problèmes ponctuels, ce document donne une vision globale de nos connaissances $»$.

Cela fait du monde..., pour ainsi dire tous les citoyens ! Cette énumération presque exhaustive peut paraître longue, mais elle montre bien tout l'intérêt que peuvent représenter des valeurs de référence de ce type dans un pays, et témoigne aussi de l'esprit humaniste et de la volonté pédagogique qui animaient l'auteur principal de l'ouvrage.

Elle se terminait par la notation suivante : "Cet ouvrage est la première mise à jour d'un document qui continuera à être revu et complété périodiquement afin de tenir compte du progrès des connaissances et de l'évolution des modes de vie."

Cela s'est révélé exact, puisque cette année 2000 verra enfin, après 3 années de préparation, élaboration, discussion, relecture..., la parution de l'édition suivante des ANC.

Mais outre l'étendue des retombées potentielles que cette initiative d'instituer des ANC français pouvait avoir, et le tournant historique qu'elle représentait en termes de santé publique, la liste d'utilisateurs... et d'utilisations qui y était mentionnée, et qui reste tout à fait valable, témoignait aussi de la nécessité de disposer de normes en matière d'apports nutritionnels. Ces normes peuvent prendre des formes ou remplir des fonctions différentes : elles peuvent par exemple être de nature réglementaire et concerner l'étiquetage, et ce sont en Europe les apports journaliers recommandés ou AJR. Les ANC sont des valeurs de référence plus complètes, parce qu'adaptées à chaque âge, aux deux sexes et aux états physiologiques particuliers, comme la grossesse ou l'allaitement, ou les activités 
musculaires intenses et régulières. Mais il existe aussi d'autres valeurs de référence, en particulier celles indiquant les limites au-delà desquelles il peut y avoir risque d'excès : ce sont les limites de sécurité. Plus que des notions différentes, il s'agit en réalité d'une échelle de valeurs.

Valeurs de référence : définitions et liste

\section{Les apports journaliers recommandés ou AJR (figure 1) et les valeurs françaises de " restauration " (tableau 1)}

Les AJR ne représentent pas à proprement parler des recommandations mais des valeurs moyennes utilisées pour l'étiquetage des denrées alimentaires : on devrait donc de ce fait plutôt les appeler " valeurs de référence étiquetage ". Ce sont les quantités de vitamines et éléments minéraux figurant dans la directive européenne relative à l'étiquetage nutritionnel (90/496/CEE), transposée dans le droit français par le décret n 931130 du 27 septembre 1993 et l'arrêté du 3 décembre 1993 (tableau 1). Elles sont issues du groupe d'Helsinki qui réunissait en 1988 des scientifiques et des représentants des professions, et sont passées dans le droit français en 1993.

Les valeurs qui avaient fait l'objet d'un avis de la commission d'étude des produits destinés à une alimentation particulière (ou CEDAP) sur la restauration des aliments en vitamines et minéraux (avis $\mathrm{n}^{\circ}$ 16 du 10 avril 1997) étaient dérivées des AJR, mais aussi des ANC français en ce qui concerne, pour la classe des vitamines, le cas du beta-carotène, qui y figure de façon identifiée (tableau 1).

\section{Les apports nutritionnels conseillés ou ANC}

Les apports nutritionnels conseillés ou ANC sont conçus pour couvrir les besoins d'une population (figure 1) : ils sont d'ailleurs nommés " apports de référence pour une population ", ou ARP, dans la traduction française (1994) du rapport européen du Comité scientifique pour l'alimentation humaine [2] de 1992. Ils sont calculés à partir de la valeur des besoins physiologiques qui, eux, sont mesurés sur un groupe d'individus, et correspondent aux " besoins moyens » ou BM du même CSAH. En ajoutant deux écarts types, pour tenir compte de la variabilité interindividuelle existant dans toute population, à ces besoins de nature individuelle, on obtient les ANC, qui sont donc de nature statistique et sont censés couvrir en théorie les besoins de $97,5 \%$ de la population.

L'écart type utilisé dans ce calcul est fixé à $15 \%$ de la valeur moyenne des besoins ; il est sensiblement plus faible que l'écart type des apports alimentaires réels en nutriments correspondants, observé dans les enquêtes de consommation, et qui se situe généralement entre 20 et $40 \%$. Cela tient au fait que les besoins physiologiques des individus présentent une dispersion plus faible que celle des apports alimentaires, beaucoup plus complexe, parce qu'elle intègre de multiples composantes, dont le choix du consommateur et la composition des aliments.

Il découle de cette définition que lorsque le niveau d'apport moyen en un nutriment donné dans une population se trouve à $77 \%$ des ANC (c'est-à-dire mathématiquement à l'ANC moins deux écarts types, donc au niveau des besoins), la loi des probabilités fait qu'un individu sur deux dans cette population est en dessous des besoins, et par conséquent $50 \%$ de la population. Cette proportion se monte à 84 $\%$ lorsque la moyenne est à $66 \%$ des ANC. Mais si l'apport moyen est équivalent à l'ANC, les besoins de la population sont théoriquement couverts. 
Il faut déjà remarquer que, comme on peut le constater à l'examen du tableau 1, des valeurs de références $[1,2]$ définies de la même manière, telles que les ANC et les ARP, peuvent, issues d'instances différentes, représenter des niveaux très différents ; de même, on s'aperçoit qu'entre 1992 et 2000, les valeurs des ANC français se sont modifiées, parfois à la hausse, comme pour la vitamine C, mais plus souvent à la baisse, comme pour plusieurs vitamines du groupe B. On voit donc que les ANC ont une valeur et relative et provisoire et ne valent que sur une période donnée. Ils nécessitent d'être réévalués, réajustés ou confirmés à intervalles réguliers, d'abord à la lumière de toutes les données scientifiques nouvelles apparues entre temps sur le sujet, qui comportent en particulier maintenant davantage d'études statistiques rigoureuses. D'autres concepts prennent également de plus en plus d'importance dans les analyses et les décisions, ce sont les dimensions de prévention et de santé publique. Enfin, il faut tenir compte de l'évolution des comportements individuels, qui introduit des différences dans les apports, en particulier en énergie, en fonction des dépenses ou des modes de vie en général, et par conséquent aussi des besoins en vitamines.

\section{Place des ANC dans l'échelle des valeurs de référence (figure 1)}

Les ANC (et le besoin moyen dont ils découlent) ne constituent pas les seules références permettant de juger de la situation nutritionnelle d'une population. Différents repères ont été fixés sur la courbe des niveaux d'apport, notamment dans l'introduction du rapport européen sur les substances nutritives du CSAH (1992-1994). En récapitulant, on dispose en tout de quatre ou cinq repères :

- le seuil d'apport minimal ou SAM du CSAH [3], qui est censé représenter en fait le seuil de la déficience ou la limite d'apparition des symptômes cliniques de la carence. Ex : $10 \mathrm{mg}$ par jour pour la vitamine C ou $100 \mu \mathrm{g}$ par jour pour l'acide folique (vitamine B9) ; en l'absence de ces données, on peut utiliser le besoin moyen moins deux écarts types ;

- le besoin moyen (BM du CSAH [3], et dietary reference intakes ou DRI [3] des Américains dans la version recommended intakes for individuals), déjà défini plus haut comme valeur de base dans le calcul des ANC : c'est donc la valeur des besoins des individus, établie selon le cas [in ANC 2000] sur la base de données métaboliques (par exemple à partir du degré d'absorption, cas de la vitamine B12) ou biochimiques (cas des vitamines B1 et B2), ou d'études épidémiologiques permettant de croiser des valeurs alimentaires et biologiques (cas de la vitamine $\mathrm{C}$ et des folates) ;

- les apports de référence pour une population (ARP du CSAH et ANC) ou besoins moyens (BM) + deux écarts types, qui intègrent donc les marges statistiques de sécurité de " couverture des besoins » : si la moyenne ou la médiane se situe à leur valeur, 97,5\% de ladite population a théoriquement ses besoins couverts (voir plus haut). Remarque : les ANC 2000 ont acquis une dimension supplémentaire par rapport aux précédents : ils sont fixés en principe comme des besoins optimaux, qui intègrent à la fois les marges statistiques définies ci-dessus et des éléments de prévention individuelle (cas des vitamines $E$ et $C$ et de l'acide folique, in ANC 2000).

- les limites de sécurité : elles représentent la valeur d'apport qui tient compte d'une marge de sécurité entre la dose la plus faible présentant des effets secondaires indésirables (lowest observed adverse effect level ou LOAEL) et les ANC, et ne concernent qu'un nombre limité de vitamines. Elles ont été établies et entérinées en 1995 par le Conseil supérieur d'hygiène publique de France (tableau 2). 


\section{Les limites de sécurité}

Étant donné que leur intégration aux recommandations est de plus en plus étendue, il est peut-être utile de bien les situer. Elles ont acquis une valeur normative dans certains pays, comme les États-Unis, qui ont ces dernières années réexaminé les recommandations. Et si, en Europe, le risque de dépasser certaines limites en ingestion quotidienne de vitamines avait déjà été mentionné dans le rapport du CSAH de 1992-1994 (tableau 4), la France avait été en 1995 la seule, avec l'avis du Conseil supérieur d'hygiène (CSHPF) [4], à statuer sur ce sujet (tableaux 3 et 4). Et, actuellement, elle semble adopter une position encore plus prudente concernant l'ensemble des vitamines hydrosolubles et des minéraux que celle prise précédemment dans l'avis du conseil d'hygiène : il s'agit de codifier les règles générales d'ajout de ces nutriments aux aliments afin de ne pas dépasser pour les plus forts consommateurs des niveaux qui pourraient conduire à terme à des risques (tableau 3).

Aux États-Unis, certaines vitamines seulement sont placées dans le tableau comportant des tolerable intake levels ou UL [3], les valeurs indiquées étant graduées selon l'âge et le sexe, au même titre que les apports conseillés eux-mêmes ; elles valent pour les vitamines $D, B 6$, l'acide folique et la niacine : les limites pour l'adulte de 19 à 70 ans, sont respectivement de $50 \mu \mathrm{g}, 100 \mathrm{mg}, 1000 \mu \mathrm{g}$ et $35 \mathrm{mg}$ par jour.

D'après la place qu'occupent les ANC, parmi les autres valeurs de référence, on peut conclure pratiquement que :

1) les ANC représentent, compte tenu des données scientifiques existantes au moment où ils sont établis, un optimum d'apport pour une population. II en découle que, pour un individu donné, l'idéal est de se situer pour chaque nutriment à une valeur d'apport comprise entre 0,8 et 1 fois les ANC [6] ;

2) les apports recommandés en général sont, et demeurent, malgré la meilleure prise en compte des données scientifiques, une notion évolutive qui devrait tendre vers toujours plus de précision(s) et d'adaptation aux réalités de la consommation et de la composition des produits. De nombreux pays s'y emploient, dont le nôtre, mais il faut aussi se rendre compte que cette réalité devient de plus en plus difficile à cerner car elle change et s'internationalise de plus en plus rapidement (produits transformés surtout). Ce souci de perfectionnisme scientifique et l'évolution des comportements et des produits expliquent pourquoi les apports conseillés ont du mal à voir le jour dans certains pays et que la phase de discussion préalable à la décision soit si longue et si laborieuse.

\section{Les données internationales}

Les apports conseillés (tableau 5)

Quand on considère les recommandations des différents pays occidentaux en ce qui concerne les apports en vitamines, on est frappé, sinon dérouté par les écarts existant entre les positions : elles ont pourtant été l'aboutissement de réflexions et d'analyses dont la pertinence et le bien-fondé sont incontestables. Ces divergences tiennent à différentes raisons qu'il est intéressant de comprendre pour y apporter des améliorations dans la mesure du possible.

Cependant, elles tendent à s'atténuer si l'on en juge par l'évolution des valeurs conseillées : il est certain qu'il se produit insensiblement, au cours de la phase d'élaboration de ces recommandations, des transferts d'informations entre pays ou experts, et que ces derniers recherchent une 
harmonisation des recommandations, nécessaire au niveau des échanges, qu'ils soient de nature commerciale ou scientifique, ou qu'ils relèvent de la politique de santé publique. De fait, les utilisateurs, de tous les horizons possibles, apprécient d'avoir des valeurs analogues lorsqu'ils ont par exemple à comparer les résultats d'études issues d'autres pays ou qu'ils sont chargés d'élaborer des produits à l'exportation...

Un autre facteur qui joue en faveur d'une relative convergence des positions concerne les résultats scientifiques portant sur l'effet bénéfique de certains nutriments, qu'ils soient donnés sous forme ajoutée ou présents naturellement dans les aliments. C'est le cas pour les facteurs alimentaires ou nutritionnels disposant d'un pouvoir anti-oxydant parallèlement à la mise en lumière du rôle des radicaux libres, que des hypothèses récentes ont fait apparaître comme des facteurs de risque de maladies dégénératives et des processus du vieillissement, lorsqu'ils sont en excès, alors qu'ils demeurent physiologiquement et " immunitairement " indispensables... II y a parfois là un effet de mode en faveur d'une relative nouveauté, qui peut mener à une certaine précipitation dans les études d'intervention et entraîner une inflation plus ou moins généralisée dans les valeurs des recommandations... Or, il est difficile parfois d'y voir clair, lorsque beaucoup de travaux, de modes différents (cellulaires, expérimentaux chez l'animal, ou épidémiologiques chez l'homme) sont en cours et n'ont pas encore révélé de résultats probants et indiscutables. Si l'on reprend l'exemple des facteurs anti-radicalaires, il est certain que les vitamines possédant un pouvoir anti-oxydant (vitamine $\mathrm{E}$, vitamine $C$ et carotènes) sont précisément en abondance dans les catégories d'aliments qui apparaissent souvent comme préventives de grandes pathologies, telles que cancers ou maladies cardio-vasculaires, à savoir les légumes et les fruits. Mais, avant de conclure à l'efficacité prépondérante de tel ou tel nutriment caractérisé et d'en majorer les apports, il faut considérer que ces aliments contiennent aussi un grand nombre de facteurs qui peuvent être au moins aussi bénéfiques et qui existent sous de multiples formes : les fibres, les polyphénols, les autres caroténoïdes, dont les rôles sont loin d'être connus.

C'est pourquoi, avant de remettre en cause ou de réajuster des recommandations d'apports qui ont au moins valeur d'usage, il convient de vérifier la validité des outils utilisés ou d'en rechercher de nouveaux.

Une politique d'apports en nutriments est en définitive la résultante de la position des experts, au regard des données scientifiques, et de l'utilisation qui en est faite ensuite par les responsables de la politique de l'alimentation.

\section{Les limites de sécurité}

II y a une prise de conscience de plus en plus générale dans les pays industrialisés de la notion de limites supérieures d'apport (upper limits des experts américains), bien que cette notion recouvre des niveaux et des formes très différents selon les pays, et, en particulier que la marge de sécurité envisagée entre la dose la plus faible qui donne des effets secondaires en ingestion chronique (LOAEL) et la limite d'emploi autorisée puisse être très variable. S'il est probable que, dans un certain nombre de cas, en particulier aux États-Unis, ou à l'échelle de l'Union européenne, une pression commerciale non négligeable joue sur le choix final des décideurs politiques par rapport aux positions des experts, il est sûr que d'autres facteurs entrent en ligne de compte, par exemple ceux liés à des particularités nationales. En France, par exemple, les consommateurs attachent une importance affective au caractère "naturel " des produits alimentaires, qui va de l'engouement pour les produits dits 
biologiques à la consommation grandissante des produits de terroir. Il est probable que le consommateur français ira, dans l'ensemble, plus spontanément vers des produits à « teneur garantie " ou " naturellement riches en... » que vers des aliments qu'il pourrait considérer comme " manipulés ", voire "trafiqués " (sic), comme les aliments enrichis ; le Français a le sentiment que plus la nourriture est traditionnelle, meilleure elle est... II a en outre une méfiance instinctive pour les dogmes scientifiques quand il ne les comprend pas et il admettra plus facilement une marge d'incertitude quant à une insuffisance éventuelle, mais aléatoire, en certaines vitamines que certaines populations plus crédules ou plus timorées quant à leur santé, vue au travers de leur alimentation. C'est ainsi que les Français sont, en tout cas à l'heure actuelle, moins consommateurs de compléments vitaminiques que les citoyens américains ou allemands [7] et réservent cette pratique aux périodes de fatigue ou de stress dans $65 \%$ des cas, ou de risque d'infection; et, contrairement aux Américains, cette consommation est le plus souvent temporaire, sous forme de cures, et abandonnée semble-t-il à l'âge de la retraite [8] !

\section{Réponses à l'insuffisance d'apport : les formes et les politiques de supplémentation}

\section{L'enrichissement}

L'enrichissement est, selon le Codex Alimentarius " l'adjonction à un aliment de un ou plusieurs éléments nutritifs essentiels qui sont ou non normalement contenus dans cet aliment, à l'effet de prévenir ou de corriger une carence démontrée en un ou plusieurs éléments nutritifs dans la population ou dans des groupes spécifiques de population $»$.

On voit que le concept d'enrichissement doit s'appliquer lorsqu'il apparaît des risques de carence démontrés dans toute ou parties d'une population : il s'agit donc d'une mesure de santé publique, mais sa justification peut varier d'un pays à l'autre en fonction de situations nutritionnelles soit conjoncturelles, soit permanentes, et des seuils de risque définis par les autorités de chaque pays. II est par conséquent là encore relatif...

\section{La restauration (ou restitution)}

Cette notion est également définie par le Codex : « c'est l'adjonction à un aliment du ou des éléments nutritifs qui ont été inévitablement perdus lors de l'application de bonnes pratiques de fabrication ou de l'entreposage et des procédures normales de manutention ". L'avis de la CEDAP en était très proche, mais avait limité cette restitution : 1) aux nutriments qui étaient contenus dans l'aliment à une teneur supérieure à $5 \%$ des AJR par $100 \mathrm{Kcal}$ et, 2) à des doses n'excédant pas $200 \%$ de la valeur initiale pour les vitamines, à l'exception du rétinol, qui en était exclu. Certains considèrent qu'il s'agit en réalité d'un enrichissement ; mais la " restauration » en diffère, tout au moins dans la conception française, par le fait que l'aliment concerné doit contenir déjà une quantité non négligeable de la vitamine à ajouter, et que cet ajout est limité à une valeur " naturelle ". Les aliments dits " à teneur garantie ", le lait UHT par exemple, sont d'un usage courant dans notre pays, mais celui-ci demeure relativement isolé concernant cet usage, pourtant bien cadré, comme on l'a vu, et qui assure au consommateur un apport en éléments nutritifs conforme à ce qu'il attend du produit, dans sa " naturalité ». Certains de nos voisins (Espagne et Belgique par exemple), ne sont d'ailleurs pas sourds à ce type d'arguments... 
Malgré le grand nombre de produits enrichis dont les fabricants demandent l'autorisation de vente en France en alimentation courante, les autorités sanitaires sont restées réticentes dans ce domaine, bien que la commercialisation des céréales enrichies soit autorisée à destination des enfants et adolescents, considérés comme un groupe particulier. Cette réticence était due essentiellement à une certaine prudence et à l'attente d'informations sur l'existence et l'importance des insuffisances d'apport, bases d'une politique d'enrichissement (voir le texte du Codex). Par ailleurs, la prudence imposait aussi de veiller à ce qu'un trop grand nombre de produits enrichis, avec des teneurs excessives en micro-nutriments, et ceux-ci même souvent redondants, ne puisse être consommé par les mêmes individus et entraîner ainsi un apport trop élevé en certains éléments. De même, comme il est très justement indiqué dans un ouvrage en cours d'édition [9], un des reproches fréquemment adressés aux aliments enrichis, comme aux compléments alimentaires, c'est d'atteindre surtout les groupes de population qui en ont le moins besoin, compte tenu de leur style de vie et du surcoût, répercuté sur le prix de vente, que représentent les investissements en recherche et en contrôle qu'ils occasionnent au fabricant. Enfin, dans le même ouvrage [9], il est souligné que "la confiance dans l'expertise a suffisamment été ébranlée dans le domaine de la sécurité alimentaire en général pour que les scientifiques fixent à un très haut niveau de qualité les preuves scientifiques appuyant les effets revendiqués ».

C'est pourquoi, c'est en toute confiance que les Français peuvent considérer l'autorisation, prise récemment et destinée à répondre aux exigences de la santé publique, d'enrichir en vitamine $D$, et avec des teneurs limitées, certaines catégories d'aliments (laits et laitages frais), les experts ayant considéré que les risques d'insuffisance en cette vitamine dans notre population pouvaient entraîner des conséquences néfastes [10].

Parallèlement, les autorités de l'Agence française de sécurité sanitaire des aliments (AFSSA) réfléchissent à des modalités d'enrichissement qui respectent les limites de sécurité (LAM, tableaux 3 et 4) pour les plus forts consommateurs tout en permettant de rehausser les plus faibles à des niveaux approchant les ANC de manière plus satisfaisante.

Les politiques d'enrichissement en général

D'une manière générale, trois éléments entrent en ligne de compte dans le choix et l'utilisation potentielle des recommandations :

- l'attitude du consommateur (méfiance, critique, recherche du « vrai »...), indissociable de son degré d'instruction et d'information et donc de l'éducation nutritionnelle qu'il a reçue ou cherché à acquérir, ce qui est de plus en plus le cas ;

- le lobbying industriel et commercial ;

- la politique alimentaire des responsables chargés de préparer les décisions et qui se fondent plus ou moins selon les pays sur des principes de santé publique : en fonction du niveau et de la validité des connaissances scientifiques du moment qu'ils sont en charge de jauger, ils peuvent choisir des marges de risques dans le sens d'une insuffisance relative, s'ils préfèrent opter pour la marge d'incertitude, ou au contraire, refuser la marge d'incertitude dans le sens sécuritaire, qui se traduit par une recommandation excédentaire, mais risquer alors des excès. C'est le compromis, ou plutôt une alliance 
bien pesée entre ces deux extrêmes qui définit une saine politique alimentaire en matière nutritionnelle.

Mais plus précisément, lorsque sont mis en évidence des problèmes d'insuffisance relevant de mesures de santé publique, il existe globalement plusieurs solutions :

* l'enrichissement fortement recommandé (mandatory) d'aliment(s) véhicule(s). C'est la solution choisie par les États-Unis pour les folates depuis le $1^{\mathrm{er}}$ janvier 1998 (ajout de $140 \mu \mathrm{g}$ d'acide folique par $100 \mathrm{~g}$ de céréales, farines, riz...), dont il est également question en Grande-Bretagne pour la même vitamine. Une recommandation d'enrichissement de ce type existe dans de nombreux pays européens en ce qui concerne les vitamines $A$ et $D$ dans la margarine : Belgique, Irlande, Islande, Pays-Bas, Royaume-Uni, Suède, et Danemark et Portugal pour la vitamine A seulement ; Amérique du Nord : États-Unis (dans certains produits laitiers pour la vitamine D) et Canada ; et autres pays du monde industrialisé, comme l'Australie [11]. C'est ce que la France a choisi également en ce qui concerne la vitamine $D$ (voir plus haut) ;

* la campagne de sensibilisation à une alimentation plus équilibrée et plus riche dans les vitamines, ou les nutriments en général, qui paraissent insuffisants, ciblés aux besoins sur le(s) groupe(s) à risque. C'est pour l'instant ce que les pouvoirs publics français ont retenu et entrepris concernant l'acide folique chez les jeunes femmes, après avoir constaté un déficit moyen d'apport dans cette classe d'âge (tableau 6) [12]. Une campagne est initiée actuellement en France auprès des adolescentes et des jeunes femmes pour les inciter à avoir une alimentation régulièrement variée et comportant suffisamment de : 1 ) légumes et fruits, aliments riches en particulier en cette vitamine, mais aussi en carotènes, vitamines $C$ et fibres ; 2 ) protéines animales, riches en fer, de laitages, riches en calcium, de poisson, riche en iode, etc. (brochure Bien se nourrir au féminin du Comité français d'éducation pour la santé ou CFES). Avec, en perspective, les autres membres de la famille ou de l'entourage... ;

* ce qui n'exclut pas que d'autres initiatives puissent être envisagées par ailleurs, telle que l'autorisation d'aliments enrichis et ciblés sur les groupes à risque, dans les limites qui auront été fixées.

Car, pour conclure sur cette perspective, on peut dire, comme Ambroise Martin [9] que " l'enrichissement des aliments est à la fois un outil de santé publique et une voie normale d'innovation et de valorisation pour l'industrie agro-alimentaire. Ces deux aspects ne sont pas incompatibles, mais plutôt complémentaires, si un certain nombre de conditions sont réunies et de règles définies et respectées ». Et, plus loin : « ... il serait dommage qu'une modification incontrôlée des comportements détruise trop rapidement la culture alimentaire française ».

On ne saurait mieux dire, et chaque Français digne de ce nom devrait se reconnaître dans ce souhait !

\section{REFERENCES}

1. DUPIN H, ABRAHAM J, GIACHETTI I (1992). Apports nutritionnels conseillés pour la population française. CNRS/CNERNA. Paris : Tec et Doc, Lavoisierv $146 \mathrm{pp}$.

2. ANC 2000. Apports nutritionnels conseillés. Paris : Tec et Doc Lavoisier. 
3. Rapport du Comité scientifique de l'alimentation humaine (1992-1994). Substances nutritives et consommation énergétique (avis émis le 11 décembre 1992). Commission européenne. Direction générale de l'industrie. Office des publications officielles des communautés européennes. Luxembourg, 1994.

4. Conseil supérieur d'hygiène publique de France (1996). Les limites de sécurité dans les consommations alimentaires des vitamines et des minéraux. Avis du 12 septembre 1995. Paris. Tec et Doc Lavoisier.

5. POTIER DE COURCY G, MAFFRE J et le groupe de travail de la CEDAP (2000). Méthode et analyse d'une simulation de l'enrichissement des aliments en vitamines et minéraux. Document émanant du gouvernement français et adressé à la DG 24 de la Commission européenne.

6. DRI (1998). Institute of Medicine, National Research Council. Dietary reference intakes : folate, other B vitamins, and choline (1998). Washington, DC : National Academy Press.

7. Conférence de presse du CEIV, Roche, 1996.

8. VOLATIER JL. Rapport sur une enquête de consommation des compléments alimentaires en France réalisée en 1998-1999 par l'OCA (Credoc). Communication personnelle.

MARTIN A (2000). Vitamines, oligo-éléments, supplémentation diverses : intérêts et risques. In : MULTON JL. Additifs, auxiliaires technologiques dans les IAA. Paris : Tec et Doc Lavoisier.

VERGER P, GARABÉDIAN M, POTIER DE COURCY G, et al. (2000). A methodology for risk assessment of micronutrients in fortified foods. Br J Nutr (soumis).

. TNO-report (1996). Scoop task 7.1.1 working group : Scientific considerations for the development of measures on the addition of vitamin and minerals to foodstuffs. TNO Nutrition and Food Research Institute des Pays-Bas.

IFN/ANIA/I'ALLIANCE 7 (1999). L'estimation de la situation nutritionnelle de la population française. 
Illustrations

Origines et utilisations des ANC

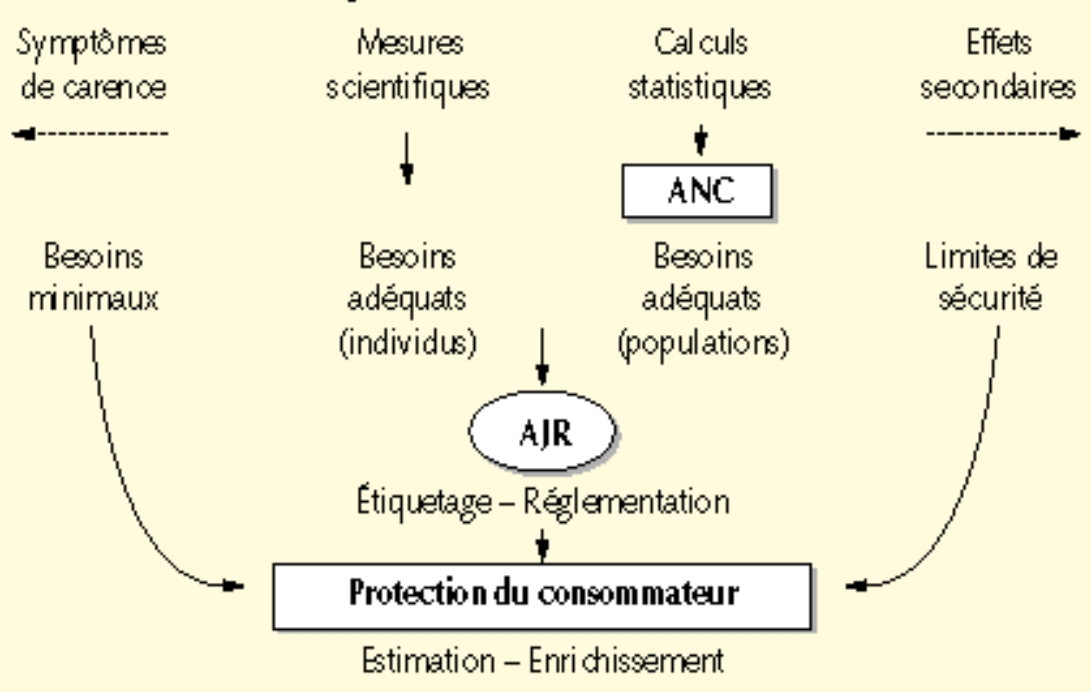

Figure 1. Place des apports conseillés dans la politique alimentaire.

\begin{tabular}{|c|c|c|c|c|c|}
\hline Vitamines & $\begin{array}{c}\text { AJR } \\
\text { CEE } 90 / 496 \\
\text { et France } \\
1993\end{array}$ & $\begin{array}{c}\text { Valeurs } \\
\text { \& restaur ation * } \\
\text { France/CEDAP } \\
1997\end{array}$ & $\begin{array}{c}\text { ARP-CSAH } \\
\text { 1992-1994 } \\
\text { Europe } \\
\text { ad ultes [3] }\end{array}$ & $\begin{array}{c}\text { ANC } 1992 \\
\text { [1] } \\
\text { France } \\
\text { adultes }\end{array}$ & $\begin{array}{l}\text { ANC } 2000 \\
\text { France } \\
\text { adultes } \\
{[2]}\end{array}$ \\
\hline$A(\mu g$ ou $E R)$ & 800 & 800 & $700-600$ & $800-1000$ & $600-800$ \\
\hline Bêta-carotène (mg) & - & 3 & - & $2,9-3,6$ & $2,4-3,2$ \\
\hline$D(\mu g)$ & 5 & 5 & $0 / 10-0 / 10$ & 10 & $5-5$ \\
\hline$E(m g)$ & 10 & 10 & $>4->3$ & 12 & $12-12$ \\
\hline $\mathrm{B} 1$ (mg) & 1,4 & 1,4 & $1,1-0,9$ & $1,6-1,8$ & $1,1-1,3$ \\
\hline B2 (mg) & 1,6 & 1,6 & $1,6-1,3$ & $1,5-1,8$ & $1,5-1,6$ \\
\hline B6 (mg) & 2 & 2 & $1,5-1,1$ & $2-2,2$ & $1,5-1,8$ \\
\hline$B 9(\mu g)$ & 200 & 200 & $200-200$ & 300 & $300-330$ \\
\hline $\mathrm{B} 12(\mu \mathrm{g})$ & 1 & 1 & $1,4-1,4$ & 3 & {$[2,4-2,4]$} \\
\hline$C(m g)$ & 40 & 60 & $45-45$ & 80 & $110-110$ \\
\hline $\mathrm{PP}$ ou niacine $(\mathrm{mg})$ & 18 & 18 & $18-14$ & $15-18^{*}$ & $11-14$ \\
\hline Biotine (mg) & 0,15 & 0,15 & - & - & $50-50$ \\
\hline Ac-pantothénique (mg) & 6 & 6 & - & $10-10$ & $5-5$ \\
\hline
\end{tabular}

Tableau 1. Valeurs nutritionnelles de référence en vitamines.

La valeur de gauche représente l'apport conseillé pour les femmes, l'autre pour les hommes. 


\begin{tabular}{|lcc}
\hline Vitamines & $\begin{array}{c}\text { Limites de sécurité } \\
\text { en plus de Yapport alimentaire }\end{array}$ & $\begin{array}{c}\text { Correspondance } \\
\text { en ANC (1992) }\end{array}$ \\
\hline Vitamine A (rétino D)(1) & $1000 \mu \mathrm{g}$ ou $3300 \mathrm{UI}$ & $\times 1$ \\
Vitamine D & $25 \mu \mathrm{g}$ ou $1000 \mathrm{UI}$ & $\times 2,5$ \\
Vitamine E & $40 \mathrm{mg}$ ou $60 \mathrm{UI}$ & $\times 4$ \\
Vitamine B6 (2) & $5 \mathrm{mg}$ & $\times 2$ \\
Niacine (vit PP) & $33 \mathrm{mg}$ & $\times 2$ \\
Acide folique(3) & $600 \mu \mathrm{gg}$ & $\times 2$ \\
Vitamine C & $1000 \mathrm{mg}$ & $\times 12$ \\
\hline
\end{tabular}

Tableau 2. Limites de sécurité concernant les vitamines (in Les limites de sécurité dans les consommations alimentaires des vitamines et des minéraux, 1996) [4]. (1) Cette limite de sécurité ne vaut pas pour le beta carotène, qui ne présente pas de risques connus aux simples doses nutritionnelles.

(2) Les vitamines $B 1, B 2$ et B12 bénéficient d'une régulation plus stricte au niveau de l'absorption que la vitamine B6.

(3) L'acide folique est très faiblemement toxique par lui-même, mais risque, à dose élevée (>= $5 \mathrm{mg} / \mathrm{j}$ ) de masquer une carence en B12, avec de possibles conséquences neurologiques irréversibles.

\begin{tabular}{|c|c|c|c|c|}
\hline Vitamines & $\begin{array}{l}\text { Limites de sécurité } \\
\text { CSHPF }\end{array}$ & $\begin{array}{l}\text { Limites de sécurité } \\
\text { complémentaires }\end{array}$ & $\begin{array}{c}\text { ANC } \\
\text { moyens utilisés }\end{array}$ & $\begin{array}{l}\text { Limites d'apport } \\
\text { maximal }\end{array}$ \\
\hline A totale $(\mu g / j)$ & & 1000 & 800 & 1800 \\
\hline -rétinol $(\mu \mathrm{g} / \mathrm{j})$ & 1000 & & 800 & 1800 \\
\hline$f$ carotène $(\mu \mathrm{g} / \mathrm{j})$ & & 6000 & 2400 & 8400 \\
\hline $\mathrm{D}(\mu \mathrm{g} / \mathrm{i})$ & 25 & 20 & 5 & 20 \\
\hline$E(m g / i)$ & 40 & & 12 & 52 \\
\hline $\mathrm{C}(\mathrm{mg} / \mathrm{D})$ & 1000 & & 80 & 1100 \\
\hline B2 (mg/j) & & 16 & 1,6 & 17,6 \\
\hline B6 (mg/j) & 5 & & 2 & 7 \\
\hline B9 $(\mu \mathrm{g} / \mathrm{j})$ & 600 & & 300 & 900 \\
\hline Niacine & 33 & & 13 & 50 \\
\hline
\end{tabular}

Tableau 3. Limites d'apport maximal (LAM) concernant les vitamines (d'après Potier de Courcy et Maffre, 2000 [5], sauf pour la niacine).

* Les limites d'apport maximal sont égales aux limites de sécurité plus la valeur moyenne (parfois arrondie) d'une fois I'ANC. 


\begin{tabular}{|c|c|c|c|}
\hline $\begin{array}{l}\text { Vitamines } \\
\text { (quantité par jour) }\end{array}$ & $\begin{array}{c}\text { Fance } \\
\text { LAM 1995-2000 }\end{array}$ & $\begin{array}{c}\text { CSAH (UE) } \\
1992-1994 \text { [3] }\end{array}$ & $\begin{array}{c}\text { États-Unis } \\
\text { DRI : UL* } 1998 \text { [6] }\end{array}$ \\
\hline A totale $(\mu \mathrm{g})$ & 1800 & - & - \\
\hline \multirow[t]{2}{*}{-rétinol $(\mu g)$} & 1800 & $\mathrm{H}: 9000$ & - \\
\hline & & F jeunes : 7500 & \\
\hline$f$ carotène $(\mu g)$ & 8400 & - & - \\
\hline$D(\mu g)$ & 20 & 50 & 50 \\
\hline$E$ (mg ou TE) & 52 & 2000 & - \\
\hline$C(\mathrm{mg})$ & 1100 & - & - \\
\hline B2 (mg) & 17,6 & - & - \\
\hline \multirow[t]{2}{*}{ B6 (mg) } & 7 & $50=$ & 35 \\
\hline & & limite de toxicité (mg) & \\
\hline B9 ou acide folique $(\mu \mathrm{g})$ & 900 & - & 1000 \\
\hline Niacine & $(50)$ & - & 15 \\
\hline
\end{tabular}

Tableau 4. Limites de sécurité dans les apports alimentaires en vitamines selon les pays : les valeurs relevées sont celles pour les adultes, et pas pour les femmes enceintes et allaitantes et les enfants.

TE : tocophérol équivalent.

* UL : Valeurs qui s'entendent avec l'alimentation, sauf pour l'acide folique et la niacine, où il s'agit des apports par compléments ou aliments enrichis. 


\begin{tabular}{|c|c|c|c|c|c|c|c|c|}
\hline & $\begin{array}{l}\mathrm{B} 1 \\
\mathrm{mg}\end{array}$ & $\begin{array}{l}\text { B2 } \\
\text { mg }\end{array}$ & $\begin{array}{c}\mathrm{B} 3 / \mathrm{PP} \\
\mathrm{mg}\end{array}$ & $\begin{array}{l}\mathrm{B} 6 \\
\mathrm{mg}\end{array}$ & $\begin{array}{l}\text { B9 } \\
\mu g\end{array}$ & $\begin{array}{l}B 12 \\
\mu g\end{array}$ & $\begin{array}{c}\mathrm{C} \\
\mathrm{mg}\end{array}$ & $\begin{array}{c}A \\
E R / \mu g\end{array}$ \\
\hline \multicolumn{9}{|l|}{ Allemag ne, Autriche, } \\
\hline Suiss akémanique 2000 & $1,2-1,0$ & $1,41,2$ & $16-13$ & $1,5-1,2$ & $400-400$ & $3-3$ & $100-100$ & $1000-8$ \\
\hline 1991 & $1,3-1,1$ & $1,7-1,5$ & $18-15$ & $1,8-1,6$ & $300-300$ & & $75-75$ & \\
\hline Belgique $1996-2000$ & $1,1-0,9$ & $1,6-1,3$ & $18-14$ & $1,7-1,2$ & $200-200$ & $1,4-1,4$ & $70-70$ & $700-60$ \\
\hline Espagne 1994-1998 & $1,2-0,9$ & $1,8-1,4$ & $20-15$ & $1,8-1,6$ & $200-200$ & $2-2$ & $60-60$ & $1000-81$ \\
\hline France 2000 & $1,3-1,1$ & $1,6-1,5$ & 1411 & $1,8-1,5$ & $330-300$ & $2,42,4$ & $110-110$ & $800-60$ \\
\hline 1992 & $1,5-1,3$ & $1,8-1,5$ & $18-15$ & $2,2-2,0$ & $300-300$ & 3,3 & $80-80$ & $1000-8$ \\
\hline \multicolumn{9}{|l|}{ Grèce = UE } \\
\hline $\begin{array}{l}\text { Irlande } 1999 \\
1983\end{array}$ & $1-0,9$ & $1,6-1,3$ & $18-15$ & - & $300-300$ & $\begin{array}{c}1,41,4 \\
3-3\end{array}$ & $60-60$ & $700-60$ \\
\hline Italie 1986-1996 & $1,2-0,9$ & $1,7-1,3$ & $18-14$ & $1,5-1,5$ & $200-200$ & $2-2$ & $60-60$ & $700-60$ \\
\hline Pays-Bas 1989 & $1 / 1,2-1$ & $1,6-1,3$ & - & $\begin{array}{c}1,3 / 1,6- \\
1 / 1,2\end{array}$ & $\begin{array}{l}200 / 300- \\
200 / 300\end{array}$ & $\begin{array}{l}2,5 / 2,6- \\
2,2 / 2,5\end{array}$ & $70-70$ & $1000-8$ \\
\hline $\begin{array}{l}\text { Pays nordiques } 1996 \\
\text { Danemark, Finlande, } \\
\text { Islande, Norvège, Suède }\end{array}$ & $1,4-1,1$ & $1,6-1,3$ & $19-15$ & $1,5-1,2$ & $300-300$ & 2,2 & $60-60$ & $1000-8$ \\
\hline \multicolumn{9}{|l|}{ Portugal = UE } \\
\hline Royaume-Uni 1991 & $1-0,9$ & $1,3-1,1$ & $17-13$ & $1,4-1,2$ & $200-200$ & $1,5-1,5$ & $40-40$ & $700-60$ \\
\hline $\begin{array}{l}\text { Union européenne (UE) } \\
\text { 1992-1994 }\end{array}$ & $1,1-0,9$ & $1,6-1,3$ & $18-14$ & $1,1-0,9$ & $200-200$ & $1,4-1,4$ & $45-45$ & $700-60$ \\
\hline États-Unis DRI* 1998 & $1,2-1,1$ & $1,3-1,1$ & $16-14$ & $1,3-1,3$ & $400-400$ & $2,42,4$ & $100-100$ & - \\
\hline RDA 1989 & $1,5-1,1$ & $1,7-1,3$ & $19-15$ & $2-1,6$ & $200-180$ & 2,2 & $60-60$ & $1000-8$ \\
\hline FAO/OMS 1998 & $1,2-1,1$ & $1,3-1,1$ & $16-14$ & $1,3-1,3$ & $400-400$ & $2,4-2,4$ & $45-45$ & $600-50$ \\
\hline
\end{tabular}

Tableau 5. Apports conseillés par jour en vitamines dans le monde (Europe, États-Unis,

FAO/OMS). Adultes des deux sexes, essentiellement entre 20 et 50 ans.

* Il s'agit des recommandations pour les individus.

La première valeur représente l'apport conseillé pour les hommes, l'autre pour les femmes. Lorsqu'il existe une fourchette de valeurs conseillées, l'intervalle est signalé par un/. En gras, les valeurs les plus récentes, lorsqu'il y a eu des modifications. 


\begin{tabular}{|c|c|c|c|c|c|c|c|}
\hline & 1 & 2 & 3 & 4 & 5 & 6 & Ensemble \\
\hline \multicolumn{8}{|l|}{ Hommes/Femmes } \\
\hline Énergie (kcaVj) & 1886 & 2869 & 1947 & 2274 & 1838 & 2802 & 2227 \\
\hline Folates $(\mu \mathrm{g} / \overline{1})$ & 312 & 350 & 253 & 242 & 202 & 276 & 270 \\
\hline \multicolumn{8}{|l|}{ Femmes $(\mathrm{N}=253)$} \\
\hline Vit. B2 (mg/i) & 1,7 & 1,9 & 1,5 & 1,6 & 1,4 & 1,5 & 1,5 \\
\hline Vit. B6 (mg/D) & 1,6 & 2 & 1,5 & 1,5 & 1,3 & 1,6 & 1,5 \\
\hline Folates $(\mu \mathrm{g} / \overline{1})$ & 304 & 335 & 241 & 237 & 196 & 247 & 250 \\
\hline$C(m g / D)$ & 100 & 83,3 & 68,1 & 70,9 & 54,7 & 51,8 & 73,1 \\
\hline$A(E R)$ & 1308 & 1750 & 1250 & 868 & 1098 & 1332 & 1214 \\
\hline$\%$ de femmes & 81 & 15,5 & 59 & 40 & 67 & 9,5 & 50 \\
\hline Áge médian (ans) & 47 & 43 & 46 & 29 & 32 & 51 & 40 \\
\hline
\end{tabular}

Tableau 6. Apports en vitamines hydrosolubles chez l'adulte en fonction de la diversité alimentaire (dégressivité de 1 à 6) OCA/CREDOC/ASPCC 1999 (ANC 2000). 